\title{
Numerical Investigation of Transition Control by Porous Surfaces in Hypersonic Boundary Layers
}

\author{
Heinrich Lüdeke ${ }^{1}$ Neil D. Sandham ${ }^{2}$ and Viola Wartemann ${ }^{1}$ \\ 1 Institut für Aerodynamik und Strömungstechnik, DLR Braunschweig \\ Lilienthalplatz 7, 38108 Braunschweig, Germany \\ heinrich.luedekeedlr.de, viola.wartemannedlr.de \\ ${ }^{2}$ University of Southampton, UK, Southampton SO17 1BJ \\ n. sandhamesoton.ac.uk
}

\begin{abstract}
Summary
The present numerical investigation of the effect of porous surfaces on transition in hypersonic boundary layers is intended to improve understanding of the physical mechanisms and to provide numerical tools for the prediction of the associated delay in transition. Direct numerical simulations are carried out by a 4th order version of the DLR-Flower code, compared with the results of linear stability theory. Good agreement of both approaches and an accurate prediction of the damping of the Mack-mode instability which is responsible for supersonic transition is demonstrated.
\end{abstract}

\section{Introduction}

In contrast to the low speed regime, disturbance evolution in hypersonic boundary layer flows occurs relatively slowly over long length scales. In the case of low level free stream disturbances and negligible surface roughness, the transition process is dominated by exponentially growing Mack modes. For these inviscid modes the boundary layer acts as a wave guide for acoustic noise, where selected frequencies are trapped and amplified, eventually leading to transition. Recently performed stability analysis and experimental studies [2] suggested that this mechanism can be delayed by using an ultrasonically absorptive surface to damp out the Mack mode [1]. Thus the first goal of transition control in high speed boundary layers is to prevent or delay the growth of Mack modes. Numerical studies of different accuracy and effort are possible, starting from stability theory coupled with standard CFD simulations of entire configurations as well as basic studies of single surface pores by DNS. All these techniques have to be verified by analytical solutions and validated by experimental data from literature. Fedorov et al.[2] presented the first linear stability results for porous surfaces, using a one-dimensional model of flow within blind (closed) pores. For the present study direct numerical simulations have been carried out by a fourth order compact finite difference version of the DLR-Flower code implemented by Stephan Enk [3]. We limit the 3D-calculations to rectangular pores so that grids match at the interface, removing interpolation as a possible 
source of error. Two- and three-dimensional geometries of the porous surface are investigated. Further simulations of a blunt cone by the second order finite volume version of Flower will be used for comparison with stability theory of the porous surface of such configurations [4].

\section{Numerical Approach}

\subsection{Direct numerical simulation}

While the basic FLOWer code solves the compressible Reynolds-averaged Navier Stokes equations on block-structured grids with second order finite volume techniques, the high-order version used in the present work [3] uses fourth-order central differencing based on standard compact finite differences. High-order compact filters that are applied at the end of each time step. Sponge-zone boundary conditions are optional to reduce reflections. For the present work we use a 6th order filter and the standard conservative form of the Euler terms. Time advancement is applied by a five-step second order Runge Kutta method. The equations solved are the compressible Navier-Stokes equations for flow of a perfect gas with density $\rho$, velocity components $u_{i}$, pressure $p$ and internal energy $e$, written in conservation law form as

$$
\begin{gathered}
\frac{\partial \rho}{\partial t}+\frac{\partial \rho u_{j}}{\partial x_{j}}=0 \\
\frac{\partial \rho u_{i}}{\partial t}+\frac{\partial \rho u_{i} u_{j}}{\partial x_{j}}+\frac{\partial p}{\partial x_{i}}=\frac{\partial \tau_{i j}}{\partial x_{j}}+f_{i} \\
\frac{\partial \rho E}{\partial t}+\frac{\partial(\rho E+p) u_{i}}{\partial x_{i}}=-\frac{\partial q_{i}}{\partial x_{i}}+\frac{\partial u_{i} \tau_{i j}}{\partial x_{j}}+g,
\end{gathered}
$$

where $E=e+u_{i} u_{i} / 2$. Forcing terms $f_{i}$ and $g$ are included in the right hand side of the equations such that a specified parallel base flow $\bar{\rho}(y), \bar{u}_{i}(y), \bar{E}(y)$ is time independent. In practice these terms are evaluated numerically within the code by computing and storing the initial residual. The equations are closed with the perfect gas law and constitutive relations for $q_{i}$ and $\tau_{i j}$ [6]. The described multiblock approach is used in the following without modification for all grids with and without pores.

\subsection{Linear stability theory}

The linear stability code of the University of Southampton, which is used for temporal stability analysis, solves the compressible Orr-Sommerfeld equations for the growth of small disturbances superimposed on a prescribed base flow. Density waves are represented as

$$
\rho=\hat{\rho}(y) \exp [i(\alpha x+\beta z-\omega t)],
$$

and similarly for other variables, where $\alpha$ and $\beta$ are real wavenumbers in the temporal stability approach and $\omega$ as a complex frequency that is the result of the analysis. 
The imaginary part $\omega_{i}$ gives the disturbance growth rate. A caret over a variable denotes an eigenfunction. A direct matrix solution method is used, with derivatives computed with a mapped Chebyshev method using a minimum of 150 collocation points. The code was validated by comparison with a number of sources in the literature, including Malik [5]. As shown in [6], the solutions for base flows that do not satisfy the Navier-Stokes equations are not unique and vary slightly depending on the method of derivation. The Orr-Sommerfeld results presented here are based on a derivation from the conservative equations (1-3). For spatial stability investigations of the blunt-cone boundary layer the DLR-NOLOT code is used which will be described in a later chapter.

\section{Grids and Initial Solutions}

For the grid generation of the study a stretching function is used in all cases that places more points near $y=0$, including cases with pores. The wall normal grid distribution is given by a sinh function with an iteratively determined stretching factor. The calculations use periodic boundary conditions in the $x$ and $z$ directions. A no-slip wall with temperature set to the wall temperature of the base flow is applied at $y=0$ and on the surfaces within the pores. At the outer boundary characteristic conditions are used. The base flow is obtained from a separate solution of the similarity equations for compressible boundary layer flow, solved by a shooting method. We consider here at Mach number $M_{\infty}=6$, Reynolds number $\operatorname{Re}=20000$, Prandtl number $\operatorname{Pr}=0.72$, ratio of specific heats $\gamma=1.4$ and an adiabatic wall boundary condition. Viscosity $\mu$ is prescribed by Sutherland's law with a constant of $110.4 \mathrm{~K}$ and a reference temperature of $216.65 \mathrm{~K}$, leading to a wall temperature of $1522.44 \mathrm{~K}$. The base profile normal co-ordinate is normalized by the displacement thickness.

\section{Results}

\subsection{Validation for a smooth wall}

Stability diagrams of the growth rate $\omega_{i}$ against the wavenumber $\alpha$ calculated by the temporal stability code are shown in Fig. 1a). The other part of the figure (Fig. 1b)) shows the spatial growth rate $\sigma$ against the dimensionless frequency $\omega$, calculated by NOLOT. The most unstable mode is the second (Mack) mode, with a maximum amplification rate near $\alpha=2.2$ and a dimensionless frequency $\omega=2.04297$. It has to be noted, that the maxima of the temporal- and spatial growth rates are not identical, but have been compared successfully by previous tests, using a gaster transformation. The first case considered is a smooth wall boundary layer that develops a Mack mode of instability with the base flow as described in the previous section. The two-dimensional Mack mode wavelength is chosen as three times the displacement thickness, giving a streamwise wavenumber of $\alpha=2 \pi / 3$. The DNS is initialized with an artifical disturbance at $t=0$ and the eigenmode is allowed to develop from this. The initial disturbance is given by an exponentially decaying 
harmonic function for the v-velocity component and the other variables initialized to the base flow condition. From Fig. 1 it can be seen that the chosen wavenumber puts this wave near the peak growth rate of the second mode. The linear stability eigenvalue for this case is $\omega=2.04297+i 0.03712$. The DNS of this case was set up using 32 points in the streamwise $x$ direction and 401 points in the wall-normal direction $y$. Fig. 2a shows the development of the natural logarithm of the root mean square of the $v$ perturbations (integrated over the $y$ direction) as the simulation proceeds and the developed shape of the Eigenmode by DNS and LST. By $t=2$ the unstable mode has emerged and grows exponentially over the remainder of the simulation. The slope of the solid line is the growth rate from linear stability theory. The agreement for the growth rate is within 0.5\%. The shape of the Mack modes in the field is shown in Fig. 3 by p-and v-contours.

\subsection{Mack mode stabilisation by porous surfaces}

Investigating the influence of two dimensional pores on the modes of the previous section results in a reduction of the porosity into spanwise grooves. Two tests are shown here for 8 and 16 pores with a constant porosity of $25 \%$ and a pore depth of $\mathrm{d}=1.0$. The grid resolution of the pores in streamwise direction is chosen with 6 cells inside the grooves which was proven to be sufficient by a grid convergence study with 12 cells inside the pores. In Fig. 4 the Mack mode development for 8 and 16 pores is shown. The growth rate for 8 pores is calculated with $51.0 \%$ of the smooth wall case and for 16 pores with $72.2 \%$. Thus the growth rates are substantially lower compared to the smooth wall case, with higher reductions for fewer grooves. The reasons for this effect are evident from Figure 3 where there is clear flow activity within the grooves. A trailing leg of alternate low and high pressure and positive and negative normal velocity moves within the grooves as the Mack mode passes above. The Mack mode is seen to be superimposed with some high-frequency content, particularly for the 8-groove case (Fig. 4a). This is not a numerical resolution effect, but is caused by sound waves emanating from the groove corners. These waves are of larger amplitude when the grooves are wider.

To confirm the assumption that the main influence on the damping is porosity at constant pore depth and not the shape of the pores, a three-dimensional testcase with 8 square pores was set up. Two slices of the flow field are shown in Fig. 5 passing through the pores and the smooth wall part respectively. The damping rate for this case is $48.1 \%$ which is similar to the damping in the two-dimensional case.

\subsection{Spatial linear stability theory at a blunt cone}

For the stability analysis at a blunt cone which is basis for future experiments with porous surfaces, the DLR code NOLOT (NOn-LOcal Transition analysis) [7] is used. For the blunt cone case a spatial local parallel analysis is performed. For this case the NOLOT code used the same wave function as the linear stability code of section 2.2 (see equation 4), but since the NOLOT code is a spatial code the wavenumbers $\alpha$ and $\beta$ are complex and the frequency $\omega$ is real. For the laminar 
basic-flow calculation, the 2nd order finite volume version of the DLR FLOWer code is used and for the validation the freestream conditions and cone geometries are adapted to the blunt cone numerical investigations of Rosenboom et al. [4] as also used by Stetson et al. [8] for their experiments. The blunt cone has a length of about $1 \mathrm{~m}$, a nose radius of $3.81 \mathrm{~mm}$ and a half angle of $7^{\circ}$. The freestream Mach number is set to $M_{\infty}=8$, the freestream unit Reynolds number to $R e_{u}=8.202 * 10^{6} 1 / \mathrm{m}$ and the freestream static temperature to $T_{\infty}=54.35 \mathrm{~K}$. Fig. 6 a shows the Mach number distribution and $6 \mathrm{~b}$ the temperature boundary-layer profile in comparison with former CFD results from [4] for the downstream position $s=0.8 \mathrm{~m}$. Only small differences can be seen near the edge of the boundary-layer, caused by code differences. Fig. 7 shows NOLOT results for the second mode instability at $s=0.8 \mathrm{~m}$ and a frequency of $120 \mathrm{kHz}$ which is the detected maximum frequency for the growth rate. In Fig. 7a the amplitude functions $\hat{\rho}(y)$ over the wall-normal coordinate $y$, normalised with the Blasius length $l_{e}$ are given. Fig. $7 \mathrm{~b}$ shows the spatial growth rate $\sigma$ versus the streamwise position $s$ whereas the maximum spatial growth location is $s \approx 0.8 \mathrm{~m}$. For further steps of this cone study, porous boundary conditions will be implemented into NOLOT and Flower and comparison of stability and DNS results as well as experimental data is foreseen.

\section{Conclusion}

A direct numerical simulation approach has been used to study hypersonic flow over a porous surface, in which the individual pores are resolved on the computational grid. The code is validated by a Mack mode in a Mach 6 smooth wall boundary layer with fixed adiabatic wall temperature. Results for spanwise grooves and quadratic pores demonstrate strong stabilization of the Mack mode instability. Except near the region of entry to the pore the flow within the pores is one-dimensional to a good approximation, supporting one of the main assumptions made in the linear stability theory. Pore shape does not seem to be a large factor: different numbers of spanwise grooves and square pores all give similar growth rates for the same porosity. Finally preliminary investigations of a blunt cone flow by stability analysis for comparison with planned future experiments are carried out.

\section{Acknowledgements}

This work was carried out while the second author was a Guest Scientist at DLR, Braunschweig. The authors would like to acknowledge the support received from Dr. J.Longo and Prof. Dr. C.-C.Rossow in setting up this visit.

\section{References}

[1] L.M. Mack: "Boundary layer linear stability theory". AGARD Special course on stability and transition of laminar flow, 1984. 
[2] A.V. Fedorov, N.D. Malmuth, A. Rasheed, H.G. Hornung: "Stabilization of hypersonic boundary layers by porous coatings". AIAA Journal 39 (4), 2001, pp 605-610.

[3] S. Enk: "Ein Verfahren höherer Ordnung in FLOWer für LES". DLR IB-124-2007/8, Institut für Aerodynamik und Strömungstechnik, Braunschweig, Germany, 2007.

[4] I. Rosenboom, S. Hein, U. Dallmann: "Influence of Nose Bluntness on Boundary Layer Instabilities in Hypersonic Cone Flows". AIAA 99-3591, 30th AIAA Fluid Dynamics Conference 28 June - 1 July, Norfolk, Virginia, 1999.

[5] M.R. Malik: "Numerical methods for hypersonic boundary layer stability". Journal of Computational Physics 86 (2), 1990, pp 376-413.

[6] N.D. Sandham, H. Lüdeke: "A numerical study of Mach 6 boundary layer stabilization by means of a porous surface". AIAA Aerospace Sciences Meeting 2009, Orlando USA, 2009.

[7] S. Hein, F. P. Bertolotti, M. Simen, A. Hanifi, D. Henningson: "Linear nonlocal instability analysis - the linear NOLOT code". DLR-IB 223-94 A56, 1994

[8] K. F. Stetson, E. R. Thompson, J. C. Donaldson, L. G. Siler: 'Laminar boundary layer stability experiments on a cone at Mach 8, Part 2: Blunt cone". AIAA Paper 84-0006, 1984

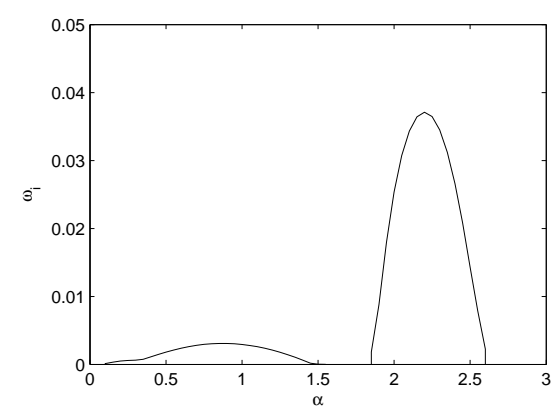

(a)

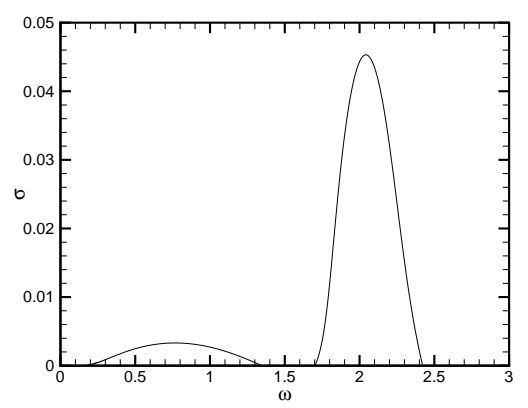

(b)

Figure 1: Stability diagrams showing the growth rate of two-dimensional disturbances as a function of (a) wavenumber $\alpha$ and (b) dimensionless frequency $\omega$, for the adiabatic wall case

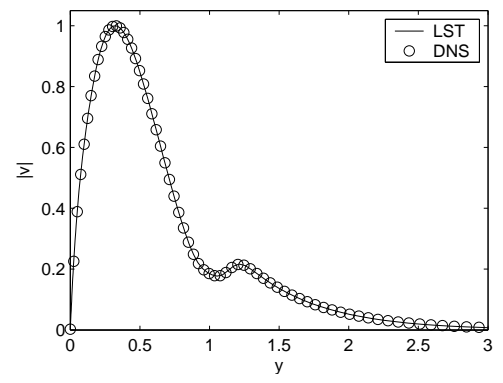

(a)

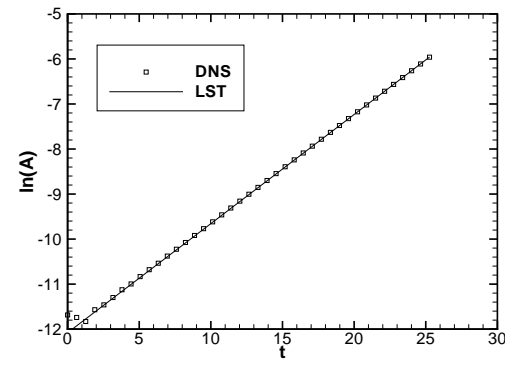

(b)

Figure 2: Smooth wall simulation of a Mack mode in an $M=6, R e=20000$ boundary layer with $T_{w}=T_{a w}$, (a) comparison of eigenfunction shape. (b) Direct numerical simulation of disturbance amplitude (symbols) and linear stability theory growth rate (solid line) 


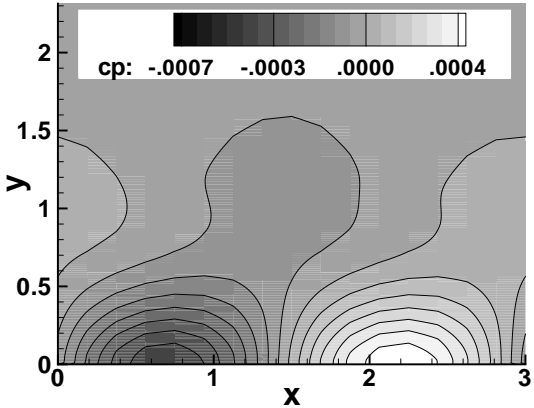

(a)

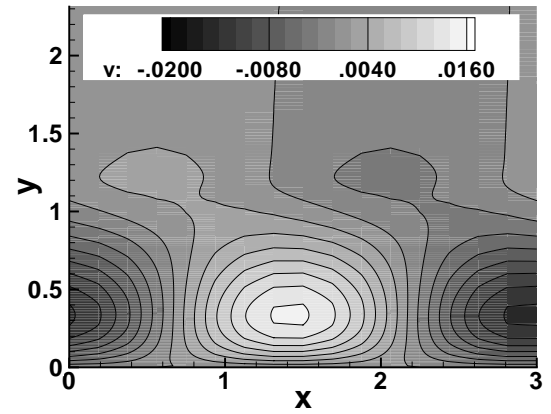

(b)

Figure 3: Mack modes developing inside a Mach 6 boundary layer at re 20000. (a) pressure contours, (b) contours of normal velocity

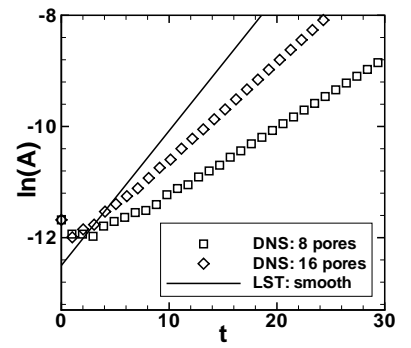

(a)

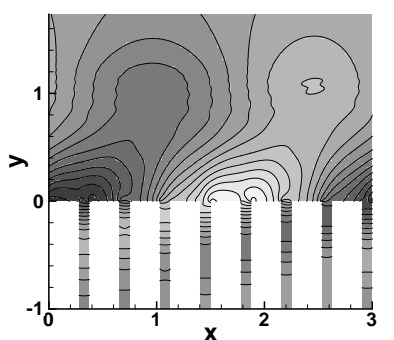

(b)

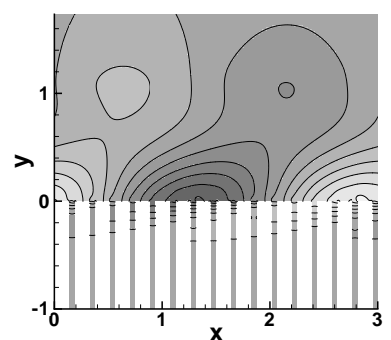

(c)

Figure 4: Mack mode developing above a porous surface with $2 \mathrm{D}$ grooves at porosity $n=0.25$ in a Mach 6 boundary layer at Re 20000. (a) temporal development, (b) contours of $\mathrm{p}$ for 8 pores and (c) for 16 pores

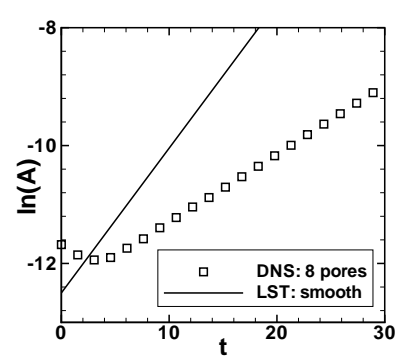

(a)

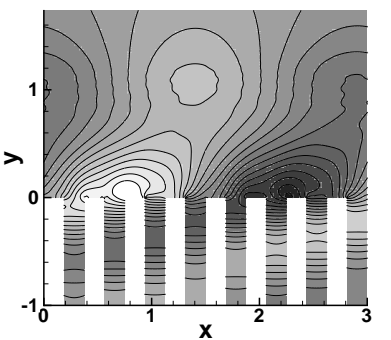

(b)

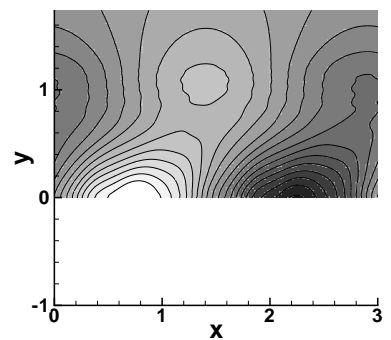

(c)

Figure 5: Mack mode developing above a porous surface with 8 quadratic pores at porosity $\mathrm{n}=0.25$ in a Mach 6 boundary layer at Re 20000. (a) temporal development, (b) contours of $\mathrm{p}$ for a plane through the pores and (c) for a plane that avoids pores 


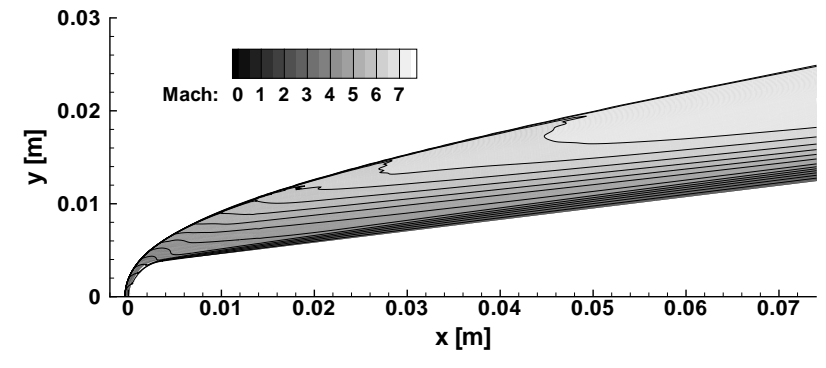

(a)

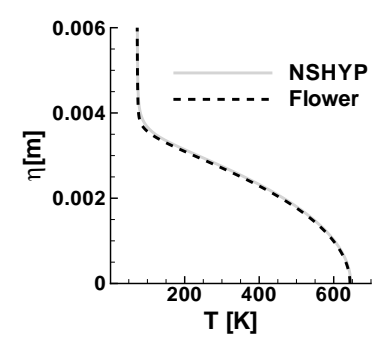

(b)

Figure 6: (a) Mach Number distribution of blunt cone at Mach 8. (b) comparison of temperature boundary layer profiles by different codes for downstream position $\mathrm{s}=0.8 \mathrm{~m}$

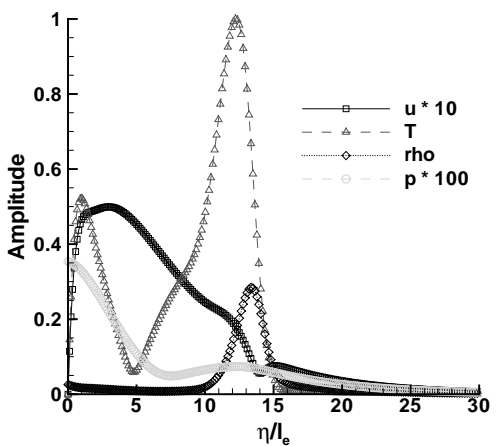

(a)

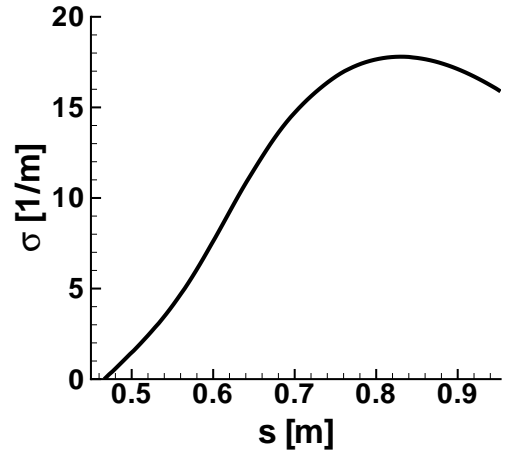

(b)

Figure 7: (a) Amplitude eigen functions of local LST for different quantities by NOLOT, (b) Growth rate $\sigma$ versus stream position $\mathrm{s}$ 